\title{
CAPACIDADE DE COORDENAÇÃO INTERORGANIZACIONAL E GERENCIAMENTO DE RISCOS
}

INTERORGANIZATIONAL COORDINATION CAPABILITY AND RISK MANAGEMENT

\section{Thiago Tomaz Luiz}

thiago t.j@hotmail.com

Programa de Pós-Graduação em Contabilidade/Universidade Federal de Santa Catarina - Florianópolis/Santa Catarina, Brasil

https://orcid.org/0000-0002-4408-3739

\section{Ilse Maria Beuren}

ilse.beuren@gmail.com

Programa de Pós-Graduação em Contabilidade/Universidade Federal de Santa Catarina - Florianópolis/Santa Catarina, Brasil

https://orcid.org/0000-0003-4007-6408

\section{Beatriz Costa Cortes}

bya_cortes1208@hotmail.com

Graduação em Ciências Contábeis/Universidade Federal de Santa Catarina - Florianópolis/Santa Catarina, Brasil https://orcid.org/0000-0001-8710-1362

\section{Resumo}

Este estudo analisa a associação da capacidade de coordenação interorganizacional com o gerenciamento de riscos do relacionamento entre startups e seu parque tecnológico. Uma pesquisa de levantamento foi realizada com gestores de startups de parques tecnológicos brasileiros. Os dados foram submetidos à análise descritiva e testes de correlação e regressão para predizer o grau de associação entre as variáveis. Os resultados denotam correlações significantes entre as variáveis do estudo e a análise da regressão desvela a capacidade de coordenação interorganizacional como antecedente do gerenciamento de riscos do relacionamento. Esses resultados elucidam a pertinência do relacionamento entre startups e seu parque tecnológico.

Palavras-chave: Capacidade de coordenação. Gerenciamento de riscos. Relacionamento interorganizacional. Startups. Parques tecnológicos.

\begin{abstract}
This study analyses the association of interorganizational coordination capability with risk management of the relationship between startups and their technology park. A survey was conducted with startups managers of Brazilian technology parks. The data were submitted to descriptive analysis and correlation and regression tests to predict the degree of association between the variables. The results show significant correlations between the study variables and the regression analysis reveals the interorganizational coordination capability as an antecedent of the relationship risk management. These results elucidate the pertinence of the relationship between the startups and its technology park.

Keywords: Coordination capability. Risk management. Interorganizational relationship. Startups. Technology parks.
\end{abstract}




\section{Introdução}

Relacionamentos interorganizacionais são fontes de recursos e de aprendizado que proporcionam vantagem competitiva e as principais razões da formação destes arranjos de negócios (Ireland, Hitt \& Vaidyanath, 2002). A formação de relações interorganizacionais ocorre pelos mais variados motivos, como a busca por recursos, suporte, conhecimento, redução de custos, entre outros. Ireland et al. (2002) observaram em seu estudo sobre alianças interorganizacionais que elas precisam ser efetivamente geridas para conseguir realizar os objetivos da parceria firmada, o que resulta em competitividade e criação de valor às organizações envolvidas.

Nesse aspecto se destacam as capacidades relacionais, como a capacidade de coordenação da relação interorganizacional, que reflete o quanto as partes buscam alinhar seus interesses, estratégias e até a tomada de decisão conjunta. A abordagem da coordenação da relação interorganizacional é importante para empresas envolvidas no relacionamento formalizado. Esta prática de gestão demonstrou impactar positivamente o desenvolvimento de novos processos em empresas taiwanesas, conforme observado no estudo de Tsou, Cheng e Wang (2019), os quais encontraram que a confiabilidade e compatibilidade entre os parceiros são fatores que afetaram a capacidade de coordenação interorganizacional.

Donada, Nogatcheswsky e Pezet (2016) destacam que os antecedentes das capacidades relacionais foram amplamente investigados, principalmente fatores internos que agem como facilitadores do desenvolvimento das capacidades, como por exemplo, rotinas, estruturas organizacionais e mecanismos de governança. Quanto aos consequentes das capacidades relacionais, ressaltam que a literatura geralmente assume relação positiva entre as capacidades relacionais e vantagem competitiva, mensurada pelo retorno na forma de desempenho financeiro e não financeiro (ex: aprendizagem, comprometimento, expectativa de continuidade da relação).

A capacidade de coordenação interorganizacional é uma forma de gestão colaborativa, caracterizada pela integração de delineamento estratégico, a tomada de decisão conjunta, entre outras características. Vivaldini (2020) discorreu sobre a gestão colaborativa associada à gestão de riscos e inferiu que estas práticas são complementares ao contribuir para a mitigação de riscos e redução de incertezas que as empresas fornecedoras têm em relação à cadeia de suprimentos.

Donada et al. (2016) apontam que estas duas práticas estão implicadas de formas distintas na relação interorganizacional. Aduzem que as empresas contam com um conjunto único de capacidades relacionais, que podem ser desenvolvidas e enfatizadas, conforme a situação exigir; enquanto os riscos geralmente são contextuais, incluem fatores internos e externos à organização. No caso da cadeia de suprimentos, as fontes de riscos da cadeia são variáveis contextuais críticas que podem ser internas ou externas à cadeia e de cada empresa que a constitui (Wagner \& Bode, 2008).

Esses resultados elucidam práticas de gestão consonantes ou divergentes na relação interorganizacional e revelam uma lacuna de pesquisa. Nessa perspectiva, presume-se que as capacidades relacionais apresentam interação com o gerenciamento de riscos interorganizacionais, um importante direcionador do desempenho da relação. Assim, tem-se a seguinte questão de pesquisa: Quais são os efeitos das capacidades relacionais e do gerenciamento de riscos interorganizacionais no desempenho do relacionamento entre startups e seu parque tecnológico? O campo da pesquisa compreende startups residentes ou associadas a parques tecnológicos e que firmaram acordo ou aliança interorganizacional para seu lançamento ou crescimento no mercado.

Apesar dos estudos sobre as empresas no contexto de parques tecnológicos serem crescentes desde a década de 1990, ainda se carece de pesquisas sobre as dinâmicas destas relações interorganizacionais (Laimer, 2015). É justamente neste ambiente que as startups se fazem mais presentes, geralmente centradas na busca pela inovação e alta competitividade. O estudo de práticas de gestão de startups segue a recente vertente da literatura gerencial destacada e observada por Samagaio, Crespo e Rodrigues 
(2018), que vem questionando e migrando da tradicional visão de que o uso de sistemas e práticas de controle restringe a postura empreendedora, dominante em startups, o que reforça a relevância do campo desta pesquisa.

Nogueira e Arruda (2016, p. 31) investigaram uma grande quantidade de startups brasileiras e constataram que "estar instalada em uma aceleradora, incubadora ou parque tecnológico representa um fator de proteção para a sobrevivência da startup; a possibilidade de ser descontinuada é de 3,45 vezes menor do que a de uma empresa instalada em escritório próprio, ou sala/loja alugada". Sugerem que tais empresas devem aproveitar as facilidades e incentivos associados ao seu processo de fundação e desenvolvimento inicial, já que estas instituições oferecem incentivos educacionais, financeiros e de relacionamento, portanto, estes espaços e vínculos podem potencializar o faturamento da empresa. Esses vínculos interorganizacionais sugerem uma gestão bastante única.

As práticas de controle gerencial devem ser prioridade em empresas que almejam superar os desafios das incertezas, como as que cercam as startups, e podem implicar no crescimento e consolidação destas no mercado (Carraro, Meneses \& Brito, 2019). O estudo também contribui com a literatura de riscos interorganizacionais em um contexto ainda pouco explorado. Embora tal literatura seja bastante desenvolvida em cadeia de suprimentos, ela pode diferir na relação investigada. Os riscos do relacionamento entre a startup e o parque tecnológico estão mais centrados em riscos relacionais, na busca pela continuidade da relação, enquanto o gerenciamento de riscos da cadeia de suprimentos, conforme Vivaldini (2020), foca eventos que podem comprometer processos de abastecimento. Outra contribuição refere-se à lacuna de pesquisa apontada em Carraro et al. (2019), as práticas de controle gerencial em startups em outros países.

\section{Desenvolvimento teórico}

\section{Capacidade de coordenação interorganizacional}

As capacidades relacionais referem-se à capacitação para integração, construção e reconfiguração de habilidades, ativos e rotinas, necessários para lidar com mudanças ambientais (Donada et al., 2016). Essas capacidades compreendem um conjunto de aptidões que favorecem as organizações criar, ampliar ou modificar sua base de recursos por meio da interação com parceiros de alianças estratégicas, além de auxiliar na criação de valor (Cordeiro \& Bataglia, 2015).

Carmeli e Azeroual (2009) destacam que as capacidades relacionais das organizações desempenham um importante papel no apoio e aquisição de conhecimento externo. As capacidades relacionais podem ser consideradas de forma multidimensional com rotinas dinâmicas (Teece, Pisano \& Shuen, 1997; Schilke \& Goerzen, 2010). Para esses autores, as dimensões das capacidades relacionais são: coordenação interorganizacional, transformação da aliança, aprendizagem interorganizacional, proatividade em alianças e portfólio de alianças.

Embora se reconheça a relevância das demais dimensões das capacidades relacionais das organizações, considera-se que a coordenação interorganizacional é primordial, pelo fato de contemplar as rotinas e processos que levam eficiência ao processo de gestão da aliança (Kumar \& Nti, 1998). Em outras palavras, a coordenação interorganizacional está voltada à eficiência com que as organizações coordenam suas atividades, alinham as estratégias e compartilham conhecimento com e entre seus aliados (Ireland et al., 2002).

A eficiência da aliança pode ser alcançada pela coordenação das interdependências entre parceiros, sincronização de atividades e conciliação dos interesses das partes relacionadas por meio de mecanismos que permitem a resolução de conflitos (Cordeiro \& Bataglia, 2015). Ressalta-se que a colaboração externa entre os membros de uma aliança apresenta diferentes benefícios. Powell, Koput e 
Smith-Doerr (1996) apontam que a colaboração pode aumentar as atividades internas de criação de valor e melhorar as vantagens competitivas, uma vez que a economia em rede é baseada no conhecimento, que não está apenas dento da organização, mas nas interações entre parceiros.

\section{Gerenciamento de riscos do relacionamento interorganizacional}

Os riscos podem estar presentes em diferentes organizações, independente do setor de atuação, objetivo econômico ou estrutura organizacional, variando quanto à forma como ocorrem ou sua intensidade (Kim \& Vonortas, 2014). A noção relativa aos riscos, geralmente está associada à consequências negativas (Wagner \& Bode, 2008). Todavia, os riscos podem ser definidos como a capacidade de uma determinada ação desencadear um efeito indesejável, que pode representar algo negativo e perigoso ou positivo, constituindo-se em uma oportunidade (Mitchell, 1995; Leitch, 2010).

Processos sistemáticos de identificação, avaliação e monitoramento de riscos representam o que é conhecido como gerenciamento de riscos (Tang, Qiang, Duffield, Young \& Lu, 2007). O gerenciamento de riscos envolve interações de várias fontes de capital e insumos, além de tecnologia, regulamentação, governança e comunicação (Gao, Sung \& Zhang, 2013). Envolve aplicação coordenada e econômica de recursos para mitigar a probabilidade ou o impacto de eventos negativos ou então para maximizar a realização de oportunidades (Hubbard, 2020).

O gerenciamento de riscos compõe-se de quatro ou cinco etapas que envolvem: classificação de riscos, identificação dos riscos, cálculo de riscos, implementação/validação de ações de gerenciamento de riscos e, às vezes, monitoramento de riscos (Lavastre, Gunasekaran \& Spalanzani, 2012). Entre os métodos utilizados no processo de gerenciamento de riscos destacam-se: planejamento de cenários, uso de painéis de especialistas, estudos Delphi e métodos de previsão (Cheng, Yip \& Yeung, 2012).

A preocupação com o gerenciamento de riscos não é apenas importante no contexto individual da organização, visto que fornece vínculo entre as organizações e o ambiente em que operam (Soin \& Collier, 2013). Ao considerar a perspectiva interorganizacional, observa-se que os riscos podem influenciar no processo de práticas para gerenciamento da cooperação entre as organizações que mantêm algum tipo de relacionamento, como por exemplo, aquelas que operam em cadeias de suprimentos (Dekker, Sakaguchi \& Kawai, 2013).

Uma maneira eficaz de gerenciamento de riscos pode estar na colaboração interorganizacional (Kim \& Vonortas, 2014). Conforme os autores, por meio de acordos formais, tais como alianças estratégicas e cooperação técnica, uma organização pode obter recursos necessários e informações valiosas para gerenciar os riscos de maneira eficaz. Ao estabelecer parcerias, os diferentes participantes podem contribuir com seus recursos na busca pela resolução de problemas que envolvem os riscos em questões relacionadas a, por exemplo, confiança e trabalho em equipe (Yeung, Chan \& Chan, 2009).

\section{Relacionamento interorganizacional entre startups e parques tecnológicos}

Os relacionamentos interorganizacionais são constituídos, mantidos e aprimorados com o propósito de levar a estratégias e objetivos de negócios que dificilmente seriam alcançados apenas por uma organização de forma individual (Cheng, 2011). Os relacionamentos interorganizacionais tendem a serem estabelecidos em torno de aspectos comuns de áreas ou tópicos de conhecimento, o que permite aprendizado incremental para todos os envolvidos nas relações (Lesser \& Prusak, 1999). Alianças interorganizacionais podem proporcionar condições superiores, o que oferece condições de diferencial e funciona como uma vantagem competitiva para empresas jovens (Kim \& Vonortas, 2014).

Em organizações que usam ou geram tecnologia em seus processos de produção é ainda mais aparente a necessidade de apoio e troca dos mais diversos insumos (Latorre, Hermoso \& Rubio, 2017). Entre as empresas jovens mais propensas a formar alianças destacam-se as startups. Elas necessitam estabelecer parcerias com universidades ou centros de pesquisas, que oferecem condições e incentivos para o 
desenvolvimento e atualizações necessárias, principalmente em relação aos aspectos que envolvem tecnologia (Ponds, Oort \& Frenken, 2010). Nesse contexto, tem-se os parques tecnológicos, que atuam com o propósito de fornecer um ambiente favorável à cooperação no processo de desenvolvimento de inovações tecnológicas (Noveli \& Segatto, 2012).

A localização das organizações em um mesmo ambiente, como proporcionado pelos parques tecnológicos, facilita aproximação e criação de relações interorganizacionais, mediação de processos de criação e compartilhamento de conhecimento e informações necessárias (Ponds et al., 2010; MontoroSánchez, Ortiz-de-Urbina-Criado \& Mora-Valentín, 2011). Os parques tecnológicos permitem que organizações neles inseridas possam compartilhar recursos, além de negociarem entre si, trabalharem projetos conjuntos e se beneficiarem das repercussões do conhecimento (Koçak \& Can, 2014).

Para as startups, os parques tecnológicos, incubadoras e aceleradoras de empresas acabam funcionando como importantes ecossistemas no processo de minimização da probabilidade de descontinuidade das atividades, ao oferecerem incentivos associados a questões de cunho educacional, financeiro e de relacionamento (Nogueira \& Arruda, 2014). Nesse ambiente, as empresas já consolidadas podem contribuir para o desenvolvimento e sobrevivência de startups (Atrasas, Dias \& Leite, 2003).

A aliança entre startups e outras organizações inseridas no contexto dos parques tecnológicos acaba por desempenhar um importante papel no processo de troca de experiências (Atrasas et al., 2003). Por meio da relação interorganizacional, as empresas menores podem superar diversos desafios tecnológicos, financeiros e operacionais (Flatten, Greve \& Brettel, 2011). A cooperação interorganizacional representa uma estratégia de economia de recursos e compartilhamento de riscos, especialmente em termos de inovação, principalmente no caso de organizações menores, que tendem a não dispor de recursos financeiros ou humanos suficientes para investir (Kofler \& Marcher, 2018).

Carraro et al. (2019) examinaram combinações de práticas de controle de gestão para auxiliar empresas startups a terem um desempenho superior. A unidade de análise da pesquisa consistiu de empresas startups estabelecidas em parques tecnológicos portugueses. Constataram que as práticas de controle gerencial utilizadas são diversas, e que, em conjunto, contribuem para o sucesso das startups, principalmente no que se refere às práticas de estratégia da organização. A partir destes achados e fundamentado na literatura, presume-se haver interações da capacidade de coordenação interorganizacional com o gerenciamento de riscos do relacionamento estabelecido entre startups e seu parque tecnológico. Assim, formula-se a seguinte hipótese de pesquisa:

$\mathbf{H}_{1}$ : A capacidade de coordenação interorganizacional está associada positivamente com o gerenciamento de riscos do relacionamento entre startups e seu parque tecnológico.

Desta maneira, entre as práticas de gestão de âmbito interorganizacional, as investigadas neste estudo referem-se à capacidade de coordenação e gerenciamento de riscos. A coordenação interorganizacional reflete o quanto a empresa consegue coordenar suas atividades, alinhar suas estratégias e compartilhar seu conhecimento com a respectiva aliança interorganizacional (Ireland et al., 2002; Tsou et al., 2019). Enquanto o gerenciamento de riscos interorganizacionais está atrelado ao desempenho da relação (Wagner \& Bode. 2008).

\section{Procedimentos metodológicos}

\section{População e coleta de dados}

Esta pesquisa de levantamento foi realizada com startups residentes ou associadas a parques tecnológicos brasileiros. Esta escolha decorre do fato dos parques tecnológicos serem ambientes formadores de clusters de inovação, o que tende a favorecer a formação de relacionamentos interorganizacionais entre empresas, universidades e órgãos públicos (Laimer, 2015). Identificaram-se 
65 parques tecnológicos brasileiros filiados à Associação Nacional de Entidades Promotoras de Empreendimentos Inovadores (Anprotec). No site de cada Parque foi consultada a lista de startups conveniadas, mas apenas 25 deles divulgaram tal informação, sendo essa a delimitação da população.

A coleta de dados ocorreu por meio de um questionário transposto na plataforma QuestionProß e

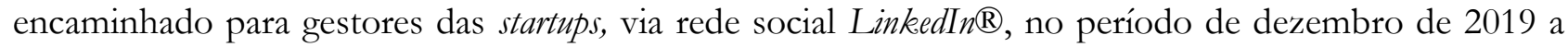
maio de 2020. Juntamente com o instrumento de pesquisa foi enviada uma carta de apresentação, com a contextualização do estudo e os contatos dos pesquisadores, para eventuais dúvidas. Dessa forma se obteve um total de 41 respostas válidas. Após análises exploratórias, não foram detectados possíveis outliers, de modo que a amostra final se concretizou em 41 startups.

\section{Variáveis do estudo}

O estudo se compõe dos construtos coordenação interorganizacional (variável independente) e gerenciamento de riscos (variável dependente). O construto coordenação interorganizacional consiste de cinco assertivas adaptadas do estudo de Tsou et al. (2019). Para o construto gerenciamento de riscos, adaptaram-se cinco assertivas do estudo de Wagner e Bode (2008). Para a mensuração destes, uma escala do tipo Likert de sete pontos foi utilizada, com os extremos ancorados em 1 = discordo fortemente e 7 = concordo fortemente.

Adicionalmente foram coletadas algumas variáveis demográficas das startups, são elas: segmento (questão aberta); cargo e tempo do gestor na empresa (questões abertas), o parque em que estão associadas (questão aberta); tempo de residência/ associação, com ponto de corte em 5 anos (binária); localização, dentro/fora do parque (binária); e porte, pelo número de funcionários (binário). Em específico para o porte, este foi mensurado de acordo com a classificação proposta pelo Serviço Brasileiro de Apoio às Micro e Pequenas Empresas (SEBRAE) (2013), na qual as micro e pequenas empresas possuem até 49 funcionários e as médias e grandes empresas apresentam mais de 50 empregados.

\section{Procedimentos para análise dos dados}

A análise dos resultados compõe-se de quatro subseções, iniciando pela análise descritiva do perfil da amostra, seguida pela análise descritiva dos construtos e itens da coordenação interorganizacional e gerenciamento de riscos, análise de correlação e análise de regressão. Para a escolha dos testes apropriados de correlação e regressão, inicialmente, no software SPSS ${ }^{\circledR} 25$, os dados foram submetidos a dois testes de normalidade: Kolmogorov-Smirnov (KS) e Shapiro-Wilk (SW). No que concerne às variáveis quantitativas, coordenação interorganizacional (KS, $p=0,20$ e $\mathrm{SW}, p=0,13)$ e gerenciamento de riscos $(\mathrm{KS}, p=0,20$ e SW, $p=0,31)$ apresentaram $p>0,05$, o que indica presença de distribuição normal dos dados para ambas variáveis. Para as variáveis demográficas (qualitativas), os dados não apresentam distribuição normal $(p<0,05)$ (Fávero \& Belfiore, 2017).

$\mathrm{Na}$ análise de correlação, utilizaram-se os coeficientes de Pearson e Spearman. O coeficiente de correlação de Pearson (@) aufere o tipo de relação linear entre duas variáveis quantitativas (Fávero \& Belfiore, 2017), como pela coordenação interorganizacional e gerenciamento de riscos. Por sua vez, o coeficiente de Spearman $\left(\mathrm{r}_{\mathrm{sp}}\right)$ é indicado como uma métrica de associação para duas variáveis qualitativas ordinais (Fávero \& Belfiore, 2017), de forma a ser apropriada para as variáveis qualitativas binárias, como porte (micro/pequena e média/grande), localização (dentro/fora do parque) e tempo de residência/associação (até 5 e mais de 5 anos). Assim, os dois testes são apresentados de forma conjunta para predizer adequadamente o grau de associação entre as variáveis do estudo.

Em acepção à análise de regressão, uma vez que as variáveis quantitativas apresentam distribuição normal, faz-se uso da regressão linear simples, no intuito de entender a relação linear entre uma variável explicativa (coordenação interorganizacional) e uma variável dependente (gerenciamento de riscos) 
(Fávero \& Belfiore, 2017). Neste sentido, a análise de regressão toma forma mediante a equação (1).

$$
y=a+\beta x+\varepsilon
$$

Em que:

y: Gerenciamento de riscos;

$x$ : Coordenação interorganizacional;

$a$ : Intercepto ou coeficiente linear;

$\beta$ : Inclinação ou coeficiente angular;

$\varepsilon$ : Termo de erro da regressão.

A instrumentalização das análises de correlação e regressão ocorreu por meio do software SPSS ${ }^{\circledR} 25$. Na sequência, a quarta seção exprime a análise dos dados, de forma coerente com o proposto nesta seção.

\section{Apresentação e análise dos resultados}

\section{Análise descritiva: caracterização das empresas do estudo}

Coerente com a própria natureza funcional e econômica, as startups da amostra atuam essencialmente com tecnologia e inovação, nos mais diversos segmentos, como agronegócios (AgTechs), educação (EdTechs), serviços financeiros (FinTechs), recursos humanos (HR Techs), esportes (Sport Techs) e outros ramos que envolvam automação, tecnologia, software, engenharia e energia. As demais características demográficas são exibidas na Tabela 1.

Tabela 1

Perfil da amostra

\begin{tabular}{|c|c|c|c|}
\hline Porte da startup (funcionários) & $n(\%)$ & Parque associada/ residente & $n(\%)$ \\
\hline Min. & 2 & BH-TEC & $1(2,4 \%)$ \\
\hline Máx. & 400 & Cietec & $1(2,4 \%)$ \\
\hline Média & 51 & Feevale Tech Park & $1(2,4 \%)$ \\
\hline Mediana & 11 & Itfetep & $1(2,4 \%)$ \\
\hline Micro/ pequena $(<50)$ & $31(75,6 \%)$ & MIDI Lages & $2(4,9 \%)$ \\
\hline Média/ grande $(\geq 50)$ & $10(24,4 \%)$ & Padetec/UFC & $1(2,4 \%)$ \\
\hline Tempo no Parque (anos) & $n(\%)$ & Pq'Tec de São Carlos & $2(4,9 \%)$ \\
\hline Min. & 0,5 & Científico e Tecnológico/ UNICAMP & $1(2,4 \%)$ \\
\hline Máx. & 18 & Tecnológico/ São José dos Campos & $9(21,9 \%)$ \\
\hline Média & 4,05 & Tecnológico Metrópole Digital & $1(2,4 \%)$ \\
\hline Mediana & 3,5 & Tecnológico/ UFMG & $1(2,4 \%)$ \\
\hline Até 5 (iniciantes) & $25(61 \%)$ & Tecnológico/ UPF & $2(4,9 \%)$ \\
\hline 5 ou mais (maduras) & $16(39 \%)$ & Porto Digital de Recife & $8(19,5 \%)$ \\
\hline Local da startup & $n(\%)$ & San Pedro Valley & $8(19,5 \%)$ \\
\hline Dentro do Parque & $28(68,3 \%)$ & Supera Parque & $1(2,4 \%)$ \\
\hline Cluster do Parque/ outro município & $13(31,7 \%)$ & Tecnosinos & $1(2,4 \%)$ \\
\hline
\end{tabular}

Fonte: Dados da pesquisa.

Acerca do porte, uma amplitude entre 2 e 400 funcionários foi encontrada. Uma vez que a média consiste em 51 e a mediana em 11 funcionários, pode-se inferir que a maior parte das startups possui menos de 51 funcionários. Isso pode ser corroborado com a porcentagem de micro e pequenas empresas $(75,6 \%)$, com até 50 funcionários. Pelo fato das startups atuarem com alto grau de tecnologia, quadros funcionais enxutos podem ser observados.

Pelo âmbito do tempo de associação/residência no parque, a amplitude concerne entre 0,5 (meio) e 18 anos. Com média e mediana de 3,5 e 4,05 respectivamente, percebe-se que a maioria das startups são relativamente novas nos parques em questão. Isso reflete em $61 \%$ estarem até 5 anos e apenas 39\% 
com permanência/associação superior a 5 anos.

Referente à localização das startups, a maior parte (68,3\%) está localizada/inserida em um parque, enquanto 31,7\% estão no cluster do parque ou em outro município. Apesar de startups de 25 parques serem constatadas, a amostra resultou em startups de 16 parques. Os parques com maior número de startups na amostra foram: Parque Tecnológico de São José dos Campos (21,94\%), Porto Digital de Recife (19,51\%) e San Pedro Valley (19,51\%). Esses, conjuntamente abrigam aproximadamente 61\% das startups analisadas.

\section{Gestão de startups associadas a parques tecnológicos}

\section{Capacidade de coordenação interorganizacional}

Conforme previamente abordado, as assertivas relativas à coordenação interorganizacional foram adaptadas do estudo de Tsou et al. (2019). Na Tabela 2 são apresentadas as assertivas, juntamente com a estatística descritiva.

Tabela 2

Análise descritiva - capacidade de coordenação interorganizacional

\begin{tabular}{c|l|c|c|c|c|c}
\hline \multicolumn{1}{c|}{ Assertivas } & Min. & Máx. & Méd. & D.P. & Med. \\
\hline $\mathbf{C I} \mathbf{1}$ & $\begin{array}{l}\text { Consultamos as sugestões do Parque Tecnológico ao tomar } \\
\text { decisões importantes. }\end{array}$ & 1 & 7 & 4,05 & 1,96 & 4,00 \\
\hline $\mathbf{C I} \mathbf{2}$ & $\begin{array}{l}\text { Temos recursos integrados para realizar um conjunto coletivo de } \\
\text { tarefas com o Parque Tecnológico. }\end{array}$ & 1 & 7 & 3,41 & 2,01 & 3,00 \\
\hline $\mathbf{C I} \mathbf{3}$ & $\begin{array}{l}\text { Combinamos recursos para realizar um conjunto coletivo de } \\
\text { tarefas com o Parque Tecnológico. }\end{array}$ & 1 & 7 & 3,54 & 1,92 & 4,00 \\
\hline $\mathbf{C I} \mathbf{4}$ & $\begin{array}{l}\text { Implantamos recursos para realizar um conjunto coletivo de } \\
\text { tarefas com o Parque Tecnológico. }\end{array}$ & 1 & 7 & 3,44 & 2,07 & 4,00 \\
\hline $\mathbf{C I} \mathbf{5}$ & $\begin{array}{l}\text { Alinhamos estratégias e compartilhamos conhecimento com o } \\
\text { Parque Tecnológico. }\end{array}$ & 1 & 7 & 4,44 & 2,03 & 5,00 \\
\hline $\mathbf{C I}$ & Construto único: média / Alpha de Cronbach = 0,927 & $\mathbf{1}$ & $\mathbf{7}$ & $\mathbf{3 , 7 8}$ & $\mathbf{1 , 7 6}$ & $\mathbf{3 , 8 0}$ \\
\hline
\end{tabular}

Legenda: Mín. = Mínimo; Máx.= Máximo; Méd. = Média; D.P. = Desvio-padrão; Med. = Mediana.

Fonte: Dados da pesquisa.

Das cinco assertivas, apenas duas (CI1 e CI5) resultaram em médias acima do ponto central da escala tipo Likert (4). Pelo contexto da mediana, a distribuição das respostas aparenta estar dividida no entorno da média teórica da escala (4). No entanto, a média e a mediana geral do construto (CI) ficaram levemente abaixo do ponto central (4), o que revela uma leve inclinação para discordância parcial, no que tange à capacidade de coordenação interorganizacional. Essa inferência está balizada em tendências pontuais (média e mediana), porém, conforme os valores mínimo e máximo encontrados, nota-se que existem startups que discordam fortemente (1) e outras que concordam fortemente (7).

Consonante à capacidade de coordenação interorganizacional entre os parques e as startups, apesar dos esforços da gestão do parque para ocorrer a aproximação e coordenação voltada ao desenvolvimento de inovações tecnológicas (Noveli \& Segatto, 2012), na percepção do gestores das startups, a capacidade de coordenação interorganizacional ainda possui espaço para ser aprimorada. Contudo, a maior parte da amostra é de startups jovens ( $<5$ anos) no parque (61\%). A coordenação interorganizacional perpassa uma construção temporal de rotinas e processos (Kumar \& Nti, 1998), o que pode demandar maior tempo para a eficiência do alinhamento entre o parque e a startup (Atrasas et al., 2003).

\section{Gerenciamento de riscos da relação interorganizacional}

O construto gerenciamento de riscos se constitui de uma adaptação do estudo de Wagner e Bode (2008). Na Tabela 3, são apresentadas as informações pertinentes à estatística descritiva dos itens e do 
construto como um todo.

Tabela 3

Análise descritiva - gerenciamento de riscos da relação

\begin{tabular}{|c|c|c|c|c|c|c|}
\hline \multicolumn{2}{|c|}{ Assertivas } & Min. & Máx. & Méd. & D.P. & Med. \\
\hline GR1 & $\begin{array}{l}\text { Em colaboração com o Parque Tecnológico, estamos } \\
\text { trabalhando na direção de um relacionamento transparente e um } \\
\text { compartilhamento aberto de informações. }\end{array}$ & 1 & 7 & 5,10 & 1,64 & 6,00 \\
\hline GR2 & $\begin{array}{l}\text { Nossa empresa elaborou planos de continuidade de negócios ou } \\
\text { de contingência que abordam vários riscos de nosso } \\
\text { relacionamento com o Parque Tecnológico. }\end{array}$ & 1 & 7 & 4,10 & 1,87 & 5,00 \\
\hline GR3 & $\begin{array}{l}\text { Monitoramos regularmente com o Parque Tecnológico sobre } \\
\text { possíveis riscos em nosso relacionamento. }\end{array}$ & 1 & 7 & 4,07 & 1,89 & 4,00 \\
\hline GR4 & $\begin{array}{l}\text { Em nossa empresa, um funcionário ou uma equipe se dedica ao } \\
\text { gerenciamento de riscos de nosso relacionamento. }\end{array}$ & 1 & 7 & 3,22 & 2,10 & 3,00 \\
\hline GR5 & $\begin{array}{l}\text { Se possível, nos protegemos de riscos relacionados ao nosso } \\
\text { relacionamento com o Parque Tecnológico. }\end{array}$ & 1 & 7 & 3,63 & 1,98 & 3,00 \\
\hline GR & Construto único: média / Alpha de Cronbach $=0,835$ & 1,4 & 6,6 & 4,02 & 1,48 & 4,00 \\
\hline
\end{tabular}

Legenda: Mín. = Mínimo; Máx.= Máximo; Méd. = Média; D.P. = Desvio-padrão; Med. = Mediana.

Fonte: Dados da pesquisa.

As maiores médias e medianas estão concatenadas a assertiva GR1, a qual se refere ao relacionamento transparente e compartilhamento aberto de informações da startup com o parque. Decorrente da percepção dos gestores das startups, infere-se que esse é um fator chave para estas empresas, o que pode implicar positivamente na superação de desafios tecnológicos, financeiros e operacionais (Flatten et al., 2011). Por sua vez, as menores médias e medianas foram obtidas em GR4, o que denota a carência de funcionários ou equipes que se dedicam ao gerenciamento de riscos do relacionamento com o parque.

No que concerne à média e mediana do construto, nota-se uma tendência central próxima ao ponto central da escala tipo Likert (4). Isso sugere um viés relativamente neutro dos gestores em relação ao gerenciamento de riscos, ou seja, por mais que alguns concordem fortemente e outros discordem fortemente, como estiva pontual geral evidencia-se que não existe discordância ou concordância acentuada. No entanto, vislumbra-se que dentro do construto gerenciamento de riscos existem aspectos que tomam diferentes ponderações para a gestão das startups. No tocante a essa discussão, não se pode desconsiderar a hipótese de que a coordenação interorganizacional pode subsidiar positivamente um eficaz gerenciamento de riscos (Kim \& Vonortas, 2014).

\section{Correlação entre as variáveis do estudo}

As análises de correlação ocorreram entre variáveis quantitativas (coordenação interorganizacional e gerenciamento de riscos) e qualitativas. As variáveis qualitativas binárias são o porte $(0=$ micro/pequena empresa e $1=$ média/grande empresa), tempo de vínculo com o parque $(0=$ até 5 anos e $1=5$ ou mais anos) e local de estabelecimento $(0=$ cluster do parque/outro município e $1=$ dentro do parque). Ambos os coeficientes (Pearson e Spearman) permitem interpretação semelhante para os resultados, no qual podem ser encontrados valores de -1 (associação linear negativa perfeita) até +1 (associação linear positiva perfeita) (Fávero \& Belfiore, 2017). Na Tabela 4 são expostas as correlações. 
Tabela 4

Correlações entre as variáveis

Painel A - Coeficiente de Pearson

\begin{tabular}{llllll}
\hline Variáveis & \multicolumn{1}{c}{$\mathbf{1}$} & $\mathbf{2}$ & $\mathbf{3}$ & $\mathbf{4}$ & $\mathbf{5}$ \\
\hline 1. Gerenciamento de riscos & 1 & & & & \\
2. Coordenação interorganizacional & $0,744^{*}$ & 1 & & & \\
3. Porte_medgde & 0,115 & 0,113 & 1 & & \\
4. Tempo_pqmais5 & 0,076 & $-0,023$ & $0,477^{*}$ & 1 & \\
5. Local_dentro & $0,493^{*}$ & $0,328^{* *}$ & $-0,345^{* *}$ & $-0,207$ & 1 \\
\hline
\end{tabular}

5. Local_dentro

\begin{tabular}{|c|c|c|c|c|c|}
\hline Variáveis & 1 & 2 & 3 & 4 & 5 \\
\hline 1. Gerenciamento de riscos & 1 & & & & \\
\hline 2. Coordenação interorganizacional & $0,758^{*}$ & 1 & & & \\
\hline 3. Porte_médio e grande & 0,094 & 0,094 & 1 & & \\
\hline 4. Tempo no parque_mais que 5 anos & 0,078 & $-0,047$ & $0,477 *$ & 1 & \\
\hline 5. Local_dentro do parque & $0,494 *$ & $0,337 * *$ & $-0,345^{* *}$ & $-0,207$ & 1 \\
\hline
\end{tabular}

Legenda: ${ }^{* *} \mathrm{p}<0,05 ;{ }^{*} \mathrm{p}<0,01$.

Nota: Significância bicaudal.

Fonte: Dados da pesquisa.

Ao percorrer as análises, resultados semelhantes podem ser identificados nos coeficientes de Pearson e Spearman. Ambos coeficientes permitem identificar cinco correlações significantes entre as variáveis do estudo. Acerca das variáveis quantitativas (coordenação interorganizacional e gerenciamento de riscos), verifica-se uma forte $\left(\varrho=0,744\right.$ e $\left.r_{\mathrm{sp}}=0,758\right)$ correlação positiva entre elas. Isso reforça que existe uma alta associação entre a coordenação interorganizacional e o gerenciamento de riscos, ou seja, existe uma relação proporcional positiva entre as duas variáveis, que tendem a crescer juntas. A congruência da coordenação interorganizacional e do gerenciamento de riscos sugere que os gestores de parques e de startups contribuem com seus recursos para a resolução de problemas que englobem os riscos em questões relacionadas para ambas (Yeung et al., 2009).

Tanto a coordenação interorganizacional $\left(\varrho=0,328\right.$ e $\left.\mathrm{r}_{\mathrm{sp}}=0,337\right)$ como o gerenciamento de riscos $\left(\varrho=0,493\right.$ e $\left.r_{\mathrm{sp}}=0,494\right)$ se correlacionam com a localização da startup. Isso denota a importância das startups estarem concentradas em um ambiente como o parque tecnológico, fato que favorece a aproximação, a capacidade de coordenação interorganizacional e o compartilhamento de informações e conhecimento (Ponds et al., 2010; Montoro-Sánchez et al., 2011). Ademais, este achado expressa que maior aproximação com o parque pode auxiliar as startups no gerenciamento de riscos, uma vez que por meio das alianças estratégicas e coordenação técnica, podem surgir informações e recursos necessários para tornar o gerenciamento de riscos eficaz (Yeung et al., 2009).

No que tange exclusivamente às variáveis qualitativas, observa-se que o porte se correlaciona positivamente com o tempo de vínculo $\left(\varrho=0,477\right.$ e $\left.\mathrm{r}_{\mathrm{sp}}=0,477\right)$ e negativamente com o local $(\varrho=-0,345$ e $\left.r_{\mathrm{sp}}=-0,345\right)$. Em linhas gerais, com o passar do tempo as empresas adquirem maturidade, o que tende naturalmente a um acréscimo no volume de negócios e, consequentemente, a um aumento do quadro funcional. Por sua vez, o fato do tempo estar negativamente associado com o local pode indicar que, à medida que as empresas vão aumentando seu quadro funcional e, desta forma, seu porte, necessitam de um espaço maior, assim deixam os locais físicos dos parques e migram para clusters de abrangência do parque ou até mesmo para outros municípios.

No entanto, como as correlações exibem medidas de associação e não de causalidade, uma possível explicação alternativa pode ser que, à medida que as empresas deixam o ambiente e espaço físico do parque para migrar ao mesmo cluster ou outro município, elas tendem a expandir seus negócios. De qualquer forma, este achado é interessante, pois demonstra a pertinência do contato entre o parque e a startup, uma vez que pode promover trocas de conhecimentos e informações e, consequentemente, acelerar o desenvolvimento e maturidade das startups (Atrasas et al., 2003; Nogueira \& Arruda, 2014). 


\section{Relação entre capacidade de coordenação interorganizacional e gerenciamento de riscos do relacionamento}

No intento de averiguar a influência da capacidade de coordenação interorganizacional no gerenciamento de riscos, uma análise de regressão linear simples foi efetuada. Na Tabela 5 são apresentadas as variáveis do modelo, os coeficientes betas (não padronizado e padronizado), o erropadrão, os valores $t$ e $p$, além do $\mathrm{R}^{2}$.

Tabela 5

Regressão Linear

\begin{tabular}{llcccccc}
\hline $\begin{array}{l}\text { Variável } \\
\text { dependente }\end{array}$ & \multicolumn{1}{c}{$\begin{array}{c}\text { Variável } \\
\text { independente }\end{array}$} & $\begin{array}{c}\text { Coef. não } \\
\text { padronizados }\end{array}$ & $\begin{array}{c}\text { Erro- } \\
\text { padrão }\end{array}$ & $\begin{array}{c}\text { Coef. beta } \\
\text { padronizado }\end{array}$ & valor- $\boldsymbol{t}$ & valor- $\boldsymbol{p}$ & $\mathbf{R}^{2}$ \\
\hline $\begin{array}{l}\text { Gerenciamento } \\
\text { de riscos }\end{array}$ & $\begin{array}{l}\text { Constante } \\
\text { Coordenação }\end{array}$ & 1,664 & 0,374 & & 4,454 & $0,000^{*}$ & 0,542 \\
\hline interorganizacional & 0,625 & 0,090 & 0,744 & 6,956 & $0,000^{*}$ & \\
\hline
\end{tabular}

Legenda: ${ }^{*} \mathrm{p}<0,01$.

Nota: $R^{2}$ ajustado.

Fonte: Dados da pesquisa.

$\mathrm{Na}$ análise de Regressão Linear Simples encontram-se subsídios para aceitar a hipótese $\mathrm{H}_{1}$, a qual estabelece que a capacidade de coordenação interorganizacional influencia positivamente $O$ gerenciamento de riscos. O coeficiente beta padronizado dessa relação é positivo $(\beta=0,744)$ e significativo $(p<0,01)$, o que demonstra a pertinência da coordenação interorganizacional como antecedente do gerenciamento de riscos. O poder de explicação $\left(\mathrm{R}^{2}\right)$ da variável dependente pela independente concerne a 54,2\%, o que indica que mais da metade da explicação do gerenciamento de riscos provém da influência direta e positiva da coordenação interorganizacional.

Coerente com este resultado, tem-se sustentação estatística para inferir que a capacidade de coordenação interorganizacional do parque com a startup pode influenciar no gerenciamento de riscos das startups. Este achado não surpreende, pois, a efetividade do gerenciamento de riscos pelas empresas tende a ser afetada positivamente pela colaboração interorganizacional (Kim \& Vonortas, 2014). Desta forma, o grau de compartilhamento de informações e de conhecimento no âmbito interorganizacional pode instigar e promover o gerenciamento de riscos (Ireland et al., 2002).

Pelo fato das startups atuarem com elevado grau tecnológico, a existência da coordenação interorganizacional configura-se como um pertinente meio propulsor da inovação tecnológica (Latorre et al., 2017). Conforme constatado nesta pesquisa, todo este contexto de capacidade interorganizacional, propiciado pelo parque, pode auxiliar no gerenciamento de riscos, inclusive, na possível mitigação dos mesmos (Hubbard, 2020). Ao entoar um olhar paralelo, uma vez que essa criação de valor se baseia em troca de conhecimento e informações, essa interação entre o parque e a startup torna-se uma fonte de vantagem competitiva para ambos (Powell et al., 1996).

Com estes achados tem-se suporte para elucidar a importância do ecossistema dos parques tecnológicos para a sobrevivência e continuidade das startups, por meio de incentivos educacionais, financeiros e de compartilhamento de informações (Nogueira \& Arruda, 2014). A troca de experiências desempenha um relevante papel para a gestão das startups (Atrasas et al., 2003), principalmente no contexto do gerenciamento de riscos (Yeung et al., 2009; Kim \& Vonortas, 2014), conforme evidenciado na discussão dos resultados desta pesquisa.

\section{Considerações finais}

Este estudo analisou a associação da capacidade de coordenação interorganizacional com o gerenciamento de riscos do relacionamento entre startups e seu parque tecnológico. Os resultados da regressão confirmaram a influência positiva postulada entre estas variáveis, o que suporta aceitar a 
hipótese de pesquisa, indicando que a capacidade de coordenação interorganizacional influencia positivamente o gerenciamento de riscos do relacionamento entre startups e seu parque tecnológico. Deste modo, conclui-se que formas de interação e colaboração interorganizacional podem promover o gerenciamento de riscos do relacionamento (Ireland et al., 2002).

A equação da regressão indica que a capacidade de coordenação explica $54,2 \%$ das variações no gerenciamento de riscos do relacionamento interorganizacional, o que sugere que mais da metade desses riscos são geridos pela coordenação interorganizacional. Isso denota impacto positivo da capacidade da empresa em coordenar o relacionamento, portanto, um consequente alternativo ao encontrado na literatura. Donada et al. (2016) ressaltam que geralmente estudos relacionam tal variável com a criação de vantagem competitiva. Assim, adiciona-se à literatura de capacidades relacionais o gerenciamento de riscos como consequente, e especula-se que, principalmente no longo prazo, o gerenciamento de riscos bem sucedido pode resultar em vantagem competitiva, ao assegurar a continuidade da relação.

No decorrer do estudo delinearam-se duas práticas de gestão interorganizacional, e apesar da relevância da coordenação interorganizacional ter sido verificada, ao confirmar sua influência no gerenciamento de riscos, ambas as práticas revelaram-se essenciais e complementares para o sucesso e longevidade do relacionamento de parceria formalizado. Argumento alinhado com as conclusões do estudo de Vivaldini (2020), que encontrou embasamento empírico para atestar a complementaridade da gestão colaborativa com a gestão de riscos de organizações na cadeia de suprimentos. Segundo o autor, estas práticas são complementares e levam à mitigação de riscos e redução das incertezas da relação.

A capacidade de coordenação interorganizacional, uma prática dos gestores/sócios das startups para alinhar, integrar e combinar estratégias e recursos para realizar tarefas coletivas com o parque, ao compartilhar conhecimento e consulta-los a tomar decisões importantes (Tsou et al., 2019), acarretou em maiores níveis de gerenciamento de riscos do relacionamento entre startups e seu parque tecnológico. Vale destacar que riscos são inerentes a todos os relacionamentos interorganizacionais, como de descumprimento de arranjos e obrigações firmadas, que provocam outro possível risco: de descontinuidade da relação. Tal tem potencial de ser definitivo para empresas em estágios iniciais, em situação de lançamento e/ou expansão de capital, como é o caso das startups. Esse resultado adiciona achados à escassa literatura de controle e gestão de relacionamentos interorganizacionais em parques tecnológicos. Dado especialmente relevante com a crescente importância de parques tecnológicos na busca por desenvolvimento econômico de sua região e país (Laimer, 2015).

O estudo também explora como algumas características das startups da amostra estão associadas às variáveis centrais de gestão da startup, com o intuito de indicar quais fatores e características das empresas estão associados às práticas de gestão investigadas. Dentre os principais achados, verificou-se que tanto a coordenação interorganizacional quanto o gerenciamento de riscos estão positivamente associadas ao fato das empresas estarem fisicamente instaladas em seu parque tecnológico, que é o caso da maioria das startups (aproximadamente 70\%). As conexões formalizadas para a colaboração entre indivíduos, universidade, organizações e pesquisa, geralmente em âmbito regional, favorecem a difusão de conhecimento local, mas com potencial de impacto maior (Ponds et al., 2010). A proximidade geográfica entre pesquisa e universidade com o mercado local traz diversas dinâmicas entre elas, formando uma rede entre indivíduos e organizações como mecanismos de spillovers difusores de conhecimento (Ponds et al., 2010). Isso revela outro benefício das empresas estarem, além de associadas, instaladas fisicamente nos parques, que é a maior capacidade de coordenação e de gerenciamento de risco interorganizacional, vantagens ainda não identificadas em estudos anteriores.

Apresentam-se avanços na literatura gerencial, a qual inicialmente buscou verificar fatores contingenciais que influenciam as escolhas e o uso de práticas de controle e gestão. Inova-se em relação às variáveis investigadas em conjunto, ao identificar a influência da capacidade de gerir o relacionamento (coordenação interorganizacional) com maior ênfase no gerenciamento de riscos do 
relacionamento, o que pode determinar o sucesso da relação e contribuir ao amadurecimento e consolidação da startup, variáveis geralmente investigadas de modo desassociado, apesar de já terem se demonstrado direcionadoras de desempenho superior da relação e ainda foram pouco exploradas na literatura interorganizacional.

Carraro et al. (2019) investigaram diversas práticas de controle gerencial para o alto desempenho de startups estabelecidas em parques tecnológicos de Portugal, e destacaram a importância da combinação de práticas de controle e gestão para diversas finalidades, dentre elas, apontaram a gestão de riscos da startup. $\mathrm{O}$ presente estudo expande a lente de investigação no que se refere à unidade de análise investigada, por um lado, o foco ateve-se em apenas duas práticas de gestão, mas a análise refere-se à gestão interorganizacional. Deste modo, tanto a literatura gerencial quanto as organizações investigadas devem avançar em direção a priorizar e identificar modos para desenvolver e enfatizar a constituição de tais capacidades, no intuito de manter e obter resultados prolíferos, como destacado por Cordeiro e Bataglia (2015), para auxiliar no alcance da eficiência da aliança pela coordenação das interdependências e conciliação dos interesses das partes relacionadas.

Estes achados podem auxiliar os gestores de startups, no sentido de priorizar e colocar em prática estratégias que visem desenvolver maior capacidade de coordenação com o propósito de assegurar a continuidade da relação. Como destacado por Samagaio et al. (2018), ao encontrar práticas e sistemas de gestão e condições que permitem a adoção das práticas em startups, considerando que empresas caracterizadas como startups geralmente têm recursos financeiros mais escassos, estudos e achados sobre a sua gestão podem auxiliar seus gestores, que são altamente empreendedores e dispostos a tomar decisões estratégicas quanto aos controles adotados, decisões associadas ao seu crescimento (Samagaio et al., 2018).

Este estudo apresenta diversas limitações. Primeiramente, se sustenta em uma quantidade limitada de startups, apenas do contexto brasileiro, com suas especificidades, como por exemplo, os incentivos fiscais no país e o papel social dos parques tecnológicos, geralmente atrelado ao desenvolvimento de uma região e triangulação com universidades locais. Abordaram-se apenas duas práticas de gestão, a capacidade de coordenação e o gerenciamento de riscos, dentre inúmeras possibilidades. Estudos futuros podem verificar quais são os drivers das capacidades relacionais existentes em parcerias desta natureza. Neste espectro, faz-se oportuna a investigação de reflexos de outras capacidades relacionais no gerenciamento de riscos interorganizacionais motivados pela inovação, como o de empresas associadas com seus parques tecnológicos, empresas incubadas com suas respectivas incubadoras e alianças destinadas à pesquisa e desenvolvimento.

Nesta linha, estudos futuros podem aumentar o escopo desta pesquisa, ao investigar de maneira conjunta os reflexos do construto multidimensional das capacidades relacionais destacado por Teece et al. (1997) e Scholke e Goerzen (2010), que irá viabilizar uma compreensão de forma mais holística sobre quais capacidades também auxiliam à gestão de riscos interorganizacionais, para tal, além da capacidade de coordenação interorganizacional, pode-se abordar a transformação da aliança, aprendizagem interorganizacional, proatividade em alianças e portfólio de alianças.

\section{Referências}

Atrasas, A. L., Dias, J. D. S., \& Leite, L. D. S. (2003). Parques tecnológicos e incubadoras de empresas: fatores de desenvolvimento tecnológico e regional em Portugal e Espanha: Relatório de viagem internacional. Embrapa Recursos Genéticos e Biotecnologia-Documentos (INFOTECA-E). Recuperado em 22 de julho de 2020, de https://www.infoteca.cnptia.embrapa.br/bitstream/doc/185038/1/doc096.pdf

Carmeli, A., \& Azeroual, B. (2009). How relational capital and knowledge combination capability enhance the performance of work units in a high technology industry. Strategic Entrepreneurship Journal, 3(1), 85-103. 
Carraro, W. B. W. H., Meneses, R., \& Brito, C. (2019). Combinação de categorias de práticas de controle de gestão para o alto desempenho de start-ups. RBGN. Revista Brasileira de Gestão de Negócios, 21(4), 861-878.

Cheng, J. H. (2011). Inter-organizational relationships and information sharing in supply chains. International Journal of Information Management, 31(4), 374-384.

Cheng, T. C. E., Yip, F. K., \& Yeung, A. C. L. (2012). Supply risk management via guanxi in the Chinese business context: The buyer's perspective. International Journal of Production Economics, 139(1), 313.

Cordeiro, J. R., \& Bataglia, W. (2015). Investigação da relação entre a capacidade relacional e o desempenho da carteira de alianças no segmento de laboratórios farmacêuticos brasileiros. Contextus Revista Contemporânea de Economia e Gestão, 13(2), 36-60.

Dekker, H. C., Sakaguchi, J., \& Kawai, T. (2013). Beyond the contract: Managing risk in supply chain relations. Management Accounting Research, 24(2), 122-139.

Donada, C., Nogatchewsky, G., \& Pezet, A. (2016). Understanding the relational dynamic capabilitybuilding process. Strategic Organization, 14(2), 93-117.

Fávero, L. P., \& Belfiore, P. (2017). Manual de análise de dados: estatística e modelagem multivariada com

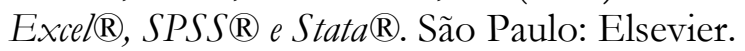

Flatten, T. C., Greve, G. I., \& Brettel, M. (2011). Absorptive capacity and firm performance in SMEs: The mediating influence of strategic alliances. European Management Review, 8(3), 137-152.

Gao, S. S., Sung, M. C., \& Zhang, J. (2013). Risk management capability building in SMEs: A social capital perspective. International Small Business Journal, 31(6), 677-700.

Hubbard, D. W. (2020). The failure of risk management: Why it's broken and how to fix it. Nova York: John Wiley \& Sons.

Ireland, R. D., Hitt, M. A., \& Vaidyanath, D. (2002). Alliance management as a source of competitive advantage. Journal of Management, 28(3), 413-446.

Kim, Y., \& Vonortas, N. S. (2014). Managing risk in the formative years: Evidence from young enterprises in Europe. Technovation, 34(8), 454-465.

Koçak, Ö., \& Can, Ö. (2014). Determinants of inter-firm networks among tenants of science technology parks. Industrial and Corporate Change, 23(2), 467-492.

Kofler, I., \& Marcher, A. (2018). Inter-organizational networks of small and medium-sized enterprises (SME) in the field of innovation: a case study of South Tyrol. Journal of Small Business \& Entrepreneurship, 30(1), 9-25.

Kumar, R., \& Nti, K. O. (1998). Differential learning and interaction in alliance dynamics: A process and outcome discrepancy model. Organization Science, 9(3), 356-367.

Laimer, C. G. (2015). Determinants of interorganizational relationships in science and technology parks: theoretical and empirical evidence. Gestão \& Regionalidade, 31(91), 122-137.

Latorre, M. P., Hermoso, R., \& Rubio, M. A. (2017). A novel network-based analysis to measure efficiency in science and technology parks: the ISA framework approach. The Journal of Technology Transfer, 42(6), 1255-1275.

Lavastre, O., Gunasekaran, A., \& Spalanzani, A. (2012). Supply chain risk management in French companies. Decision Support Systems, 52(4), 828-838.

Leitch, M. (2010). ISO 31000: 2009-The new international standard on risk management. Risk Analysis, 30(6), 887-892. 
Lesser, E., \& Prusak, L. (1999). Communities of practice, social capital and organizational knowledge. Information Systems Review, 1(1), 3-10.

Mitchell, V. W. (1995). Organizational risk perception and reduction: A literature review. British Journal of Management, 6(2), 115-133.

Montoro-Sánchez, A., Ortiz-de-Urbina-Criado, M., \& Mora-Valentín, E. M. (2011). Effects of knowledge spillovers on innovation and collaboration in science and technology parks. Journal of Knowledge Management, 15(6), 948-970.

Nogueira, V., \& Arruda, C. (2014). Causas da mortalidade de startups brasileiras. Núcleo de Inovação e Empreendedorismo, Fundação Dom Cabral. Recuperado em 22 de julho de 2020, de http://acervo.ci.fdc.org.br/AcervoDigital/Artigos $\% 20$ FDC/Artigos $\% 20 D O M \% 2025 /$ Causas $\% 20$ da $\%$ 20mortalidade $\% 20$ das $\% 20$ startups $\% 20$ brasileiras.pdf

Noveli, M., \& Segatto, A. P. (2012). Processo de cooperação universidade-empresa para a inovação tecnológica em um parque tecnológico: evidências empíricas e proposição de um modelo conceitual. RAI Revista de Administração e Inovação, 9(1), 81-105.

Ponds, R., Oort, F. V., \& Frenken, K. (2010). Innovation, spillovers and university-industry collaboration: an extended knowledge production function approach. Journal of Economic Geography, 10(2), 231-255.

Powell, W. W., Koput, K. W., \& Smith-Doerr, L. (1996). Interorganizational collaboration and the locus of innovation: Networks of learning in biotechnology. Administrative Science Quarterly, 41(1), 116145.

Samagaio, A., Crespo, N. F., \& Rodrigues, R. (2018). Management control systems in high-tech startups: An empirical investigation. Journal of Business Research, 89, 351-360.

Schilke, O., \& Goerzen, A. (2010). Alliance management capability: an investigation of the construct and its measurement. Journal of Management, 36(5), 1192-1219.

Serviço Brasileiro de Apoio às Micro e Pequenas Empresas - SEBRAE. (2013). Definição de porte de estabelecimentos segundo o número de empregados. Recuperado de: https://m.sebrae.com.br/Sebrae/Portal\%20Sebrae/UFs/SP/Pesquisas/MPE_conceito_empregados.p df

Soin, K., \& Collier, P. (2013). Risk and risk management in management accounting and control. Management Accounting Research, 24(2), 82-87.

Tang, W., Qiang, M., Duffield, C. F., Young, D. M., \& Lu, Y. (2007). Risk management in the Chinese construction industry. Journal of Construction Engineering and Management, 133(12), 944-956.

Teece, D. J., Pisano, G., \& Shuen, A. (1997). Dynamic capabilities and strategic management. Strategic Management Journal, 18(7), 509-533.

Tsou, H. T., Chen, J. S., \& Wang, Z. Q. (2019). Partner Selection, Interorganizational Coordination, and New Service Development Success in the Financial Service Industry. Canadian Journal of Administrative Sciences, 36(2), 231-247.

Vivaldini, M. (2020). Gestão colaborativa e gestão de risco: um estudo sobre capacidades complementares. Revista Gestão \& Conexões, 9(2), 120-144.

Wagner, S. M., \& Bode, C. (2008). An empirical examination of supply chain performance along several dimensions of risk. Journal of Business Logistics, 29(1), 307-325.

Yeung, J. F., Chan, A. P., \& Chan, D. W. (2009). Developing a performance index for relationshipbased construction projects in Australia: Delphi study. Journal of Management in Engineering, 25(2), 59-68. 